\title{
Twenty-Year Changes in Mortality Rates and Underlying Causes of Death in Patients with Rheumatoid Arthritis-Associated Interstitial Lung Disease, 1999-2018
}

\author{
Jinfang Gao \\ Bethune Hospital Affiliated to Shanxi Medical University \\ Lei Xin \\ The Affiliated Tumor Hospital of Shanxi Medical University \\ Qianyu Guo \\ Bethune Hospital Affiliated to Shanxi Medical University \\ $\mathrm{KeXu}$ \\ Shanxi Bethune Hospital \\ Gailian Zhang \\ Shanxi Bethune Hospital \\ Yanli Yang \\ Shanxi Bethune Hospital \\ Dan Ma \\ Bethune Hospital Affiliated to Shanxi Medical Hospital \\ Liyun Zhang ( 1315710223@qq.com ) \\ Da Yi Hospital Affiliated to Shanxi Medical University
}

Research article

Keywords: Rheumatoid arthritis, Interstitial lung disease, Mortality, Underlying cause of death

Posted Date: June 11th, 2020

DOI: https://doi.org/10.21203/rs.3.rs-34564/v1

License: (ㄷ) (i) This work is licensed under a Creative Commons Attribution 4.0 International License. Read Full License 


\section{Abstract}

Background: The treatment of rheumatoid arthritis (RA) has advanced considerably in the last 20 years. However, few population-based studies have assessed mortality rates and the underlying cause of death (UCD) in patients with RA and RA-associated interstitial lung disease (RA-ILD). We conducted a study to evaluate trends in mortality rates, demographic characteristics, and UCDs in patients with RA-ILD.

Methods: Through data from death certificates (1999-2018) acquired from the U.S. Centers for Disease Control and Prevention Multiple Cause of Death files, we explored trends in mortality rates and UCD for RA and RA-ILD patients. We evaluated trends in crude rates and age-standardised mortality rates (ASMR) for patients with RA and RA-ILD.

Results: In both RA and RA-ILD patients, ASMR variation trended downward over a 20-year period. The ASMR ratio of RA-ILD to RA decreased by $5.84 \%$. The ASMR for RA and RA-ILD stratified by sex or age group also trended downward. The change in the ASMR ratio of RA-ILD to RA differed between men and women, trending downward in women and upward in men. Arthropathies and ILD were the two most frequent UCDs for RA-ILD, while the most frequent UCDs for RA were arthropathies and ischaemic heart disease.

Conclusions: Although both RA and RA-ILD showed a downward trend in mortality, RA combined with ILD may reduce patient life span. Specifically, the mortality rate for RA-ILD remained relatively stable during the study period when ILD was the UCD, which suggests the necessity of devoting resources to active prevention, early diagnosis, and effective management of RA-ILD.

\section{Background}

Approximately $0.5-1 \%$ of the global population has rheumatoid arthritis (RA) (1). RA affects not only joints but also multiple organs. This illness is sometimes fatal, and usually economically burdensome for the patient. One of the most frequent extra-articular manifestations of RA is interstitial lung disease (ILD), which is a leading cause of illness and death in patients with RA (2). ILD, which is clinically significant among only $10 \%$ of ILD cases, can be detected in $60 \%$ of patients with RA by high-resolution computed tomography (HRCT) (3). The prevalence of RA-ILD has increased year by year (4). An earlier study showed that certain autoantibodies can be detected in the serum of patients without evidence of joint involvement, and airway abnormalities can be identified on HRCT in patients with circulating RA-related antibodies in the absence of arthritis (5). Interestingly, one group reported a hypothesis that the lung may represent the initiating site of autoimmunity (6). In the last 20 years, new biological agents have been developed that control RA joint inflammation and improve joint symptoms; however, changes in mortality and underlying cause of death (UCD) in patients with RA-ILD have rarely been studied. Previous studies of RA-ILD mortality were primarily based on deaths in patient cohorts (7, 8), which did not capture changes in RA-ILD mortality over time and did not reflect the true burden and trends of RA-ILD mortality and UCD in the general population. One previous study examined RA-ILD mortality based on death certificate data on a large population-based scale. However, the data predated 2010, and there has not been any research on RA-related mortality trends in recent years. Therefore, we conducted a population-based study of temporal trends in mortality and changes in UCD associated with RA and RA-ILD in the United States from 1999 to 2018.

\section{Methods}

Data were obtained from the U.S. Centers for Disease and Control Prevention National Center for Health Statistics Multiple Cause of Death files (1999-2018) (10), which were compiled from data collected by 57 vital statistics jurisdictions. All data contained in these database files are public records; therefore, institutional review board approval for this research was not required.

We used the following ICD-10 codes to identify RA: M05.0 (Felty syndrome), M05.1 (rheumatoid lung disease (J99.0*)), M05.2 (rheumatoid vasculitis), M05.3 (rheumatoid arthritis with involvement of other organs and systems), M05.8 (other seropositive rheumatoid arthritis), M05.9 (seropositive rheumatoid arthritis, unspecified), M06.0 (seronegative rheumatoid arthritis), M06.1 (adult-onset Still disease), M06.2 (rheumatoid bursitis), M06.3 (rheumatoid nodule), M06.4 (inflammatory polyarthropathy), M06.8 (other specified rheumatoid arthritis), and M06.9 (rheumatoid arthritis, unspecified). ILD was defined by corresponding ICD-10 codes, which included J84.1 (a code that combined postinflammatory pulmonary fibrosis and idiopathic pulmonary fibrosis), and M05.1 or J99.0 (rheumatoid lung disease). To standardise mortality rate comparisons for each year, we calculated the overall age-standardised mortality rate (ASMR) of RA and RA-ILD based on the estimated population distribution for the year 2000

We calculated yearly ASMRs of patients who died of RA or RA-ILD, as well as the ratio of the former to the latter. Among the abovementioned decedents, the trends of ASMR in both sexes and within each age group (0-54, 55-64, 65-74, 75-84, and > 84 years) were also summarised. To obtain rates of change and to highlight the trends in RA-ILD mortality compared with RA mortality, we divided patients according to two subperiods (1999-2003 and 2014-2018). We also examined change rates in the ratio of the RA-ILD ASMR to the RA ASMR overall, and according to gender. Finally, we ranked the top 10 UCDs according to the crude death rates found in the UCD-ICD sub-chapter.

\section{Results}

\section{Overall mortality trends}


From 1998 to 2018 , there were $50,567,774$ deaths in U.S. residents, of which 180,821 included mentions of RA on the death certificate. Of these RA deaths, 13,802 (7.6\%) were of RA combined with ILD. The average age-adjusted mortality rate for RA was 36.2 per 1,000,000 people in the general population. The average age of patients with RA-ILD mentioned in death certificates from 1999 to 2018 was 74.4 years, compared with 77.0 years for patients with RA. The average age of death in patients with RA was 74.8 years for males and 77.7 years for females, and the average age of death in patients with RA-ILD was 73.3 years for males and 75.0 years for females.

We observed a downward trend for ASMR over the course of 20 years for both the RA and RA-ILD groups (Fig. 1A). The ASMR ratio of RA-ILD to RA without ILD showed an upward trend year by year (Fig. 1B). The ASMR in the RA-ILD group decreased from 0.24 per 100,000 people during 19992003 to 0.15 per 100,000 people during $2014-2018$, for a rate of change of $-37.50 \%$; however, the rate of change for RA was - $33.62 \%$. The change in the ASMR ratio of RA-ILD to RA was - 5.84\%. The average age of death in patients with RA-ILD rose from 73.5 years in $1999-2003$ to 75.2 years in 2014-2018, whereas the average age of death in patients with RA rose from 76.4 to 77.2 years.

\section{Mortality trends by sex and age groups}

When we stratified the data set according to sex, we observed a decreasing trend in the ASMR for both men and women. For RA-ILD, the change in trend among women was greater than among men, while for RA, the trend was more significant in men than in women (Table 1). The ASMR ratio of RA-ILD to RA trended differently in men and women, with a downward trend observed among women and an upward trend observed among men. The mortality analysis according to gender and age group $(0-54,55-64,65-74,75-84$, and $>84$ years) revealed decreasing trends for all groups, though to differing degrees (Fig. 1C-D, Fig. 2, and Fig. 3). The largest reduction occurred among females and in people ages 55-64 years for RA-ILD, and among males and in people ages 65-74 years for RA (Tables 1 and 2). The change rates in the mortality ratio of RA-ILD to RA showed downward trends in people ages $0-54$ years (-46.67\%), 55-64 years (-28.71\%), and 65-74 years $(-5.03 \%)$, as well as rising trends in people ages $75-84$ years $(5.80 \%)$ and $>84$ years $(15.75 \%)$.

\begin{tabular}{|c|c|c|c|c|c|c|c|c|c|c|c|c|c|}
\hline \multirow[t]{4}{*}{ Variable } & \multicolumn{5}{|l|}{ RA-ILD } & \multicolumn{5}{|l|}{ RA } & \multicolumn{3}{|c|}{ ASMR ratio of RA-ILD to RA } \\
\hline & \multicolumn{2}{|c|}{ 1999-2003 } & \multicolumn{2}{|c|}{ 2014-2018 } & \multirow[t]{3}{*}{ change,\% } & \multicolumn{2}{|c|}{ 1999-2003 } & \multicolumn{2}{|c|}{ 2014-2018 } & \multirow[t]{3}{*}{ change, $\%$} & \multicolumn{2}{|c|}{ Ratio $\times 10^{-2}$} & \multirow[t]{3}{*}{ Change,\% } \\
\hline & Deaths & AMSR & Deaths & AMSR & & Deaths & AMSR & Deaths & AMSR & & 1999- & 2014- & \\
\hline & & & & & & & & & & & 2003 & 2018 & \\
\hline all & 3450 & 0.24 & 2950 & 0.15 & -37.50 & 48740 & 3.48 & 43959 & 2.31 & -33.62 & 6.90 & 6.49 & -5.84 \\
\hline Female & 2212 & 0.31 & 1861 & 0.17 & -45.16 & 35793 & 4.23 & 32139 & 2.95 & -30.26 & 7.33 & 5.76 & -21.37 \\
\hline Male & 1238 & 0.18 & 1089 & 0.13 & -27.78 & 12947 & 2.31 & 11820 & 1.44 & -37.66 & 7.79 & 9.03 & 15.86 \\
\hline
\end{tabular}

Table 1

Cumulative Percentage Change in ASMRs(deaths per 100,000 people) stratified by sex grouping of RA-ILD and RA, and ASMRs Ratio of RA-ILD to RA, $1999-2018$

\begin{tabular}{|c|c|c|c|c|c|c|c|c|c|c|c|c|c|}
\hline \multirow[t]{4}{*}{ Age groups } & \multicolumn{5}{|l|}{ RA-ILD } & \multicolumn{5}{|l|}{ RA } & \multicolumn{3}{|c|}{$\begin{array}{l}\text { mortality ratio of RA-ILD to } \\
\text { RA }\end{array}$} \\
\hline & \multicolumn{2}{|c|}{ 1999-2003 } & \multicolumn{2}{|c|}{ 2014-2018 } & \multirow[t]{3}{*}{ change,\% } & \multicolumn{2}{|c|}{ 1999-2003 } & \multicolumn{2}{|c|}{$2014-2018$} & \multirow[t]{3}{*}{ change,\% } & \multicolumn{2}{|c|}{ Ratio $\times 10^{-2}$} & \multirow[t]{3}{*}{ Change,\% } \\
\hline & Deaths & Rate & Deaths & Rate & & Deaths & Rate & Deaths & Rate & & 1999- & 2014- & \\
\hline & & & & & & & & & & & 2003 & 2018 & \\
\hline $0-54$ years & 170 & 0.02 & 94 & 0.01 & -50.00 & 1795 & 0.15 & 1636 & 0.14 & -6.67 & 13.33 & 7.14 & -46.47 \\
\hline $\begin{array}{l}55-64 \\
\text { years }\end{array}$ & 464 & 0.37 & 352 & 0.17 & -54.05 & 4422 & 3.46 & 4598 & 2.23 & -35.55 & 10.69 & 7.62 & -28.71 \\
\hline $\begin{array}{l}65-74 \\
\text { years }\end{array}$ & 1021 & 1.11 & 870 & 0.61 & -45.05 & 11301 & 12.27 & 10133 & 7.10 & -42.14 & 9.05 & 8.59 & -5.03 \\
\hline $\begin{array}{l}75-84 \\
\text { years }\end{array}$ & 1357 & 2.16 & 1051 & 1.46 & -32.41 & 19905 & 31.68 & 14559 & 20.24 & -36.11 & 6.82 & 7.21 & 5.80 \\
\hline >84years & 438 & 2.03 & 583 & 1.83 & -9.85 & 11317 & 52.54 & 13031 & 40.92 & -22.12 & 3.86 & 4.47 & 15.75 \\
\hline
\end{tabular}

Table 2

Cumulative Percentage Change in mortality rates(deaths per 100,000 people) stratified by age grouping of RA-ILD and RA, and mortality ratio of RAILD to RA, $1999-2018$ 


\section{Rank of UCD}

For RA-ILD, arthropathies and ILD consistently ranked among the top two causes of death in both time periods. Ischaemic heart diseases, pulmonary heart disease, and diseases of pulmonary circulation all dropped in the rankings over the course of the follow-up period. Malignant neoplasms rose in rank from fifth to third, and chronic lower respiratory diseases became slightly more frequent (Table 3). Interestingly, influenza and pneumonia dropped from the 10th to the 13th place. In RA, the top five causes of death were arthropathies, ischaemic heart disease, malignant neoplasms, chronic lower respiratory disease, and other forms of heart disease in both time periods (Table 4).

Table 3

The top 10 underlying-cause-of-death of RA-ILD

\begin{tabular}{|c|c|c|c|c|c|}
\hline \multicolumn{3}{|l|}{ 1999-2003 } & \multicolumn{3}{|l|}{ 2014-2018 } \\
\hline UCD - ICD Sub-Chapter & $\begin{array}{l}\text { UCD - ICD Sub- } \\
\text { Chapter Code }\end{array}$ & $\begin{array}{l}\text { Crude } \\
\text { Rates }\end{array}$ & UCD - ICD Sub-Chapter & $\begin{array}{l}\text { UCD - ICD Sub- } \\
\text { Chapter Code }\end{array}$ & $\begin{array}{l}\text { Crude } \\
\text { Rates }\end{array}$ \\
\hline Arthropathies & M00-M25 & 0.136 & Arthropathies $\rightarrow$ & M00-M25 & 0.097 \\
\hline $\begin{array}{l}\text { Other respiratory diseases principally } \\
\text { affecting the interstitium }\end{array}$ & J80-J84 & 0.042 & $\begin{array}{l}\text { Other respiratory diseases principally } \\
\text { affecting the interstitium } \rightarrow\end{array}$ & J80-J84 & 0.041 \\
\hline Ischaemic heart diseases & $120-125$ & 0.015 & Chronic lower respiratory diseases $\uparrow$ & J40-J47 & 0.008 \\
\hline Chronic lower respiratory diseases & J40-J47 & 0.009 & Malignant neoplasms $\uparrow$ & $\mathrm{COO}-\mathrm{C} 97$ & 0.007 \\
\hline Malignant neoplasms & $\mathrm{C} 00-\mathrm{C} 97$ & 0.008 & Ischaemic heart diseases $\downarrow$ & $|20-| 25$ & 0.006 \\
\hline Other forms of heart disease & $130-151$ & 0.006 & Other forms of heart disease $\rightarrow$ & $|30-| 51$ & 0.003 \\
\hline $\begin{array}{l}\text { Pulmonary heart disease and diseases } \\
\text { of pulmonary circulation }\end{array}$ & $126-128$ & 0.003 & Other bacterial diseases $\uparrow$ & A30-A49 & 0.002 \\
\hline Other bacterial diseases & A30-A49 & 0.003 & Cerebrovascular diseases $\uparrow$ & $160-169$ & 0.001 \\
\hline Cerebrovascular diseases & $160-169$ & 0.002 & Lung diseases due to external agents $\uparrow$ & J60-J70 & 0.001 \\
\hline Influenza and pneumonia & J09-J18 & 0.002 & Systemic connective tissue disorders $\uparrow$ & М30-М35 & 0.001 \\
\hline
\end{tabular}

Table 4

The top 10 underlying-cause-of-death of RA

\section{9-2003}

UCD - ICD Sub-Chapter

Arthropathies

Ischaemic heart diseases

Malignant neoplasms

Chronic lower respiratory diseases

Other forms of heart disease

Cerebrovascular diseases

Diabetes mellitus

Other respiratory diseases principally affecting the interstitium

Other bacterial diseases

Hypertensive diseases

\section{4-2018}

UCD - ICD Sub-Chapter CD - ICD Sub-
Chapter Code

Crude Rates

M00-M25

0.955

120-125

0.603

Arthropathies $\rightarrow$

0.324

Ischaemic heart diseases $\rightarrow$

0.194

Malignant neoplasms $\rightarrow$

Chronic lower respiratory diseases $\rightarrow$

0.180

Other forms of heart disease $\rightarrow$

0.170

Organic, including symptomatic, mental disorders $\uparrow$

$160-169$

0.076

Other degenerative diseases of the nervous system $\uparrow$

E10-E14

J80-J84

0.073

Hypertensive diseases $\uparrow$

0.073

A30-A49

0.072

110-I15

Cerebrovascular diseases $\downarrow$
UCD - ICD SubChapter Code

M00-M25

I20-I25

C00-C97

J40-J47

|30-|51

F01-F09

G30-G31

I10-I15

$160-169$

E10-E14
Crude Rates

0.679

0.307

0.306

0.194

0.139

0.111

0.101

0.095

\section{Discussion}

The findings of this study indicate that in both RA and RA-ILD, mortality showed a significant decline in the last 20 years and the presence of ILD decreased survival in patients with RA. While RA is more common in women than in men, previous studies have suggested that the prevalence of RAILD was greater among men than among women or was similar between genders. In the present study, although the ASMR of females was greater than that of males, the ASMR ratio of RA-ILD to RA was higher in men than in women, trending upwards in men and downwards in women. This 
observation suggests that the proportion of male patients with RA dying from ILD was higher than the proportion of female patients, and the proportion of male patients increased between 1999-2003 and 2014-2018. Hence, male patients with RA-ILD have an increased risk of death, and the risk has been increasing over the course of 20 years. These findings are consistent with those of previous cohort studies $(11,12)$.

Compared to other connective tissue diseases, patients with RA-ILD may be older, and exhibit a higher proportion of usual interstitial pneumonia (UIP). Owing to immunosenescence and inflammaging, the aged lung undergoes functional and structural changes that facilitate the occurrence of pulmonary fibrosis. Hence, ILD is more likely to occur in older patients with RA. In the present study, we found that the elderly accounted for the majority of patients with RA-ILD deaths, whereas RA-ILD patient mortality in the 55-64 years group showed significant reductions. Furthermore, the proportion of ILD cases increased in patients aged 55-74 years whose death certificates mentioned RA and decreased in patients aged $>74$ years. This suggests that RA-ILD survival was extended. However, the reasons accounting for this increase in life expectancy are not well characterised.

To answer this question, we conducted an analysis of UCD in RA-ILD, which indicated that arthropathies and ILD are ranked as the top two most frequent UCDs in patients with RA and ILD mentioned in death certificates. This was the case during the entire 20-year follow-up period. Surprisingly, although there was a decrease in the death rate among patients with RA-ILD mentioned in death certificates, little significant changes occurred in the mortality rate when ILD was the UCD. Therefore, the decline in RA-ILD mortality may be more due to an improvement in living standards, control of other complications, and active treatment of primary diseases than due to treatment of ILD. One possible explanation is that there is a critical unmet need with respect to the management of RA-ILD patients. There is also evidence that treatment with methotrexate in patients with RA increased the prevalence of interstitial lung disease by approximately $0.3-11 \%$ based on numerous case reports and case series $(13,14)$. Other evidence suggests that treating with abatacept versus TNF inhibitors (TNF-Is) might be associated with the occurrence of ILD in RA patients, which can lead to more severe pulmonary symptoms and even death $(15,16)$. Further prospective cohort studies are warranted to better illustrate the association or causation between TNF-Is and ILD. A longitudinal multicenter study of 68 RA-ILD patients was conducted from 2007 until 2018 in Madrid. The results showed that patients receiving rituximab were less likely to develop functional respiratory impairment than were patients treated with other therapies (17). Tozumab showed a good safety profile in patients with RA-ILD and a potential effect on the stabilisation of pulmonary manifestations. Nevertheless, large sample-size randomised controlled trials and prospective studies are needed to validate these findings. In addition, there are a lack of sensitive and specific disease predictors. Studies showed that possible predictors of mortality include lung carbon monoxide diffusion function, high IgM rheumatoid factor levels (18), and UIP patterns (19). However, these indicators are not specific or are difficult to use for the early identification of a disease. A recent study indicated that the MUC5B promoter variant was the strongest genetic risk factor. The MUC5B promoter variant was strongly correlated with the occurrence of RA-ILD and with UIP on imaging (20). Nevertheless, the clinical application of this test appears to be far off. A prospective cohort study (21) showed that there was an association between active articular RA and development of RA-ILD, and decreasing systemic inflammation might modify the natural history of RA-ILD. Therefore, more attention should be paid to active prevention, early diagnosis, and effective management of this condition.

Ischaemic heart disease is a very common UCD in patients with RA or RA-ILD. A previous population-based cohort study indicated that ischaemic heart disease and congestive heart failure were more likely to occur in the RA-ILD group than patients with RA but not ILD, with the difference being more significant for congestive heart failure ( $8.5 \%$ in the RA-ILD group and $4.4 \%$ among those with RA but not ILD). A cross-sectional cohort study of 2013 patients with RA from 21 hospitals in China showed that treatment with hydroxychloroquine (HCQ) was a protective factor against cardiovascular disease, whereas ILD, hypertension, and hyperlipidemia were independent risk factors. In our study, the UCD ranking of ischaemic heart disease in RA-ILD patients decreased from third to fifth, probably owing to the widespread use of HCQ and the effective management of ischaemic heart diseases. In this study, malignant neoplasms ranked fourth on the UCD list in RA-ILD patients during 2014-2018, and compared with 19992003 , they showed an increasing trend. In fact, certain meta-analyses have suggested that RA patients, compared with the general population, tend to have lymphomas and lung malignancies (22). RA patients with ILD and a UIP pattern were often subjected to higher mortality rates than those with other patterns and were also more likely to die of lung cancer (19). Systemic connective tissue disorders also showed an upward trend in the UCD ranking of RA-ILD, possibly because of the widespread use of various autoantibody detection techniques. Some studies indicated that infectious diseases were increasingly frequent causes of death associated with RA, and that the most common cause of death was pneumonia based on chronic ILD $(19,23)$. Our study reached similar conclusions. However, surprisingly, influenza and pneumonia dropped out of the top 10 most frequent UCDs during the study period. In contrast, the incidence of other bacterial diseases was elevated. This could be related to more attention being paid to lung infections based on ILD.

\section{Conclusions}

The mortality rates in both RA and RA-ILD showed downward trends from 1999-2003 to 2014-2018, with extended patient survival. However, RA in combination with ILD may reduce the life span of patients with RA. The ASMR was higher in women with RA-ILD than in men with RA-ILD; however, among patients whose death certificates mentioned RA, the proportion of men with ILD was higher than the proportion of women. Men and older adults are at a higher risk for RA-ILD death. Whether 20 years ago or more recently, arthropathies and ILD are the two most frequent UCDs in patients with RA and ILD mentioned in death certificates. HRCT can detect lung disorders in more than half of RA patients, and approximately $10 \%$ of these patients develop clinically significant ILD. It is difficult to identify the above patients for early intervention in the clinical course of their lung disorder, and even with early detection, available therapeutics do not have a clear beneficial effect on the prevention or delayed progress of ILD. Hence, it 
remains urgent to identify new biological markers to help diagnose and predict disease progression, to study its pathogenesis, and to develop more targeted drugs against RA-ILD.

\section{Abbreviations}

ASMR: age-standardised mortality rates; ILD: interstitial lung disease; RA: rheumatoid arthritis; RA-ILD: rheumatoid arthritis-associated interstitial lung disease; UCD: underlying cause of death

\section{Declarations}

\section{Ethics approval and consent to participate}

The ethical review was exempted because of the use of open data.

\section{Consent for publication}

Not applicable.

\section{Availability of data and materials}

The data used in this study came from a national mortality database maintained by the CDC. The analysed during the current study are available from the corresponding author on reasonable request.

\section{Competing interests}

The authors declare that they have no competing interests.

\section{Funding}

Financial support for this research was provided by the National Natural Science Foundation of China (81202356, 81771768 and 81771292$)$, Shanxi Provincial Key Research and Development Project (201803D31136), and the applied basic research project of Shanxi Science and Technology Department (201901D111416).

\section{Authors' contributions}

Jinfang Gao and Liyun Zhang conceived the study, guided the analyses, and wrote the article draft. Lei Xin, Qianyu Guo collected and analysed the data and critically revised the draft. Ke Xu and Gailian Zhang are the guarantors of the study. All authors have read and approved the manuscript, and ensure that this is the case.

\section{Acknowledgements}

The authors thank Mrs. Xiaoting Wen for the data analysis.

\section{References}

1. GE F, E N, J L, P K, R C. Methotrexate-Associated Pneumonitis and Rheumatoid Arthritis-Interstitial Lung Disease: Current Concepts for the Diagnosis and Treatment. Frontiers in medicine 2019;6:238.

2. N S, J V, BR E, JJ S, K M, TR M, et al. Impact of the pattern of interstitial lung disease on mortality in rheumatoid arthritis: A systematic literature review and meta-analysis. Seminars in arthritis and rheumatism 2019;49(3):358-365.

3. M G, MJ M, S A, ME M, MU M. Rheumatoid Arthritis Related Interstitial Lung Disease: Patterns of High-resolution Computed Tomography. Cureus 2020;12(2):e6875

4. K R, JJ S, AL O, AM K, AL C, A F, et al. Rheumatoid Arthritis-Interstitial Lung Disease in the United States: Prevalence, Incidence, and Healthcare Costs and Mortality. The Journal of rheumatology 2019;46(4):360-369.

5. Demoruelle MK, Weisman MH, Simonian PL, Lynch DA, Sachs PB, Pedraza IF, et al. Brief report: airways abnormalities and rheumatoid arthritisrelated autoantibodies in subjects without arthritis: early injury or initiating site of autoimmunity? Arthritis Rheum 2012;64(6):1756-61. 
6. Pinheiro FA, Souza DC, Sato El. A Study of Multiple Causes of Death in Rheumatoid Arthritis. J Rheumatol 2015;42(12):2221-8.

7. $\mathrm{CH}, \mathrm{O} \mathrm{H}, \mathrm{AB} P, \mathrm{SP} U, \mathrm{~A}$ L, E B, et al. A population-based cohort study of rheumatoid arthritis-associated interstitial lung disease: comorbidity and mortality. Annals of the rheumatic diseases 2017;76(10):1700-1706.

8. C H, T E, O H, E B. Rheumatoid Arthritis-Associated Interstitial Lung Disease: Clinical Characteristics and Predictors of Mortality. Respiration; international review of thoracic diseases 2019;98(5):455-460.

9. AL O, JJ S, DB S, A F, ER F, J S, et al. Rheumatoid arthritis-interstitial lung disease-associated mortality. American journal of respiratory and critical care medicine 2011;183(3):372-8.

10. Centers for Disease Control and Prevention, National Center for Health Statistics. Multiple Cause of Death (1999-2018) on CDC WONDER Online Database, released in 2020. http://wonder.cdc.gov/mcd-icd10.html (Accessed on Mar 20, 2020). In. 3.0 ed.

11. $\mathrm{C} \mathrm{H}, \mathrm{OH}, \mathrm{AB} P, \mathrm{SP} U, A \mathrm{~L}, \mathrm{E} \mathrm{B}$, et al. A population-based cohort study of rheumatoid arthritis-associated interstitial lung disease: comorbidity and mortality. Annals of the rheumatic diseases 2017;76(10):1700-1706.

12. D K, SK C, CB C, JY C, WT C, SJ H, et al. Impact of interstitial lung disease on mortality of patients with rheumatoid arthritis. Rheumatology international 2017;37(10):1735-1745.

13. Roubille C, Haraoui B. Interstitial lung diseases induced or exacerbated by DMARDS and biologic agents in rheumatoid arthritis: a systematic literature review. Semin Arthritis Rheum 2014;43(5):613-26.

14. GE F, R C, E N. Methotrexate and interstitial lung disease: controversies and questions. A narrative review of the literature. Rheumatology (Oxford, England) 2019;58(11):1900-1906.

15. EH K, Y J, RJ D, J L, JA S, SC K. Risk of exacerbation of pulmonary comorbidities in patients with rheumatoid arthritis after initiation of abatacept versus TNF inhibitors: A cohort study. Seminars in arthritis and rheumatism 2019.

16. Y H, W L, Z C, Y W, Y H, S T. Effect of tumor necrosis factor inhibitors on interstitial lung disease in rheumatoid arthritis: angel or demon? Drug design, development and therapy 2019;13:2111-2125.

17. C V, MA N, F R, L L, O S, MJ R, et al. Efficacy of rituximab in slowing down progression of rheumatoid arthritis-related interstitial lung disease: data from the NEREA Registry. Rheumatology (Oxford, England) 2020.

18. C H, T E, O H, E B. Rheumatoid Arthritis-Associated Interstitial Lung Disease: Clinical Characteristics and Predictors of Mortality. Respiration; international review of thoracic diseases 2019;98(5):455-460.

19. T K, A H, A T, K K, S N, H T, et al. Related factors, increased mortality and causes of death in patients with rheumatoid arthritis-associated interstitial lung disease. Modern rheumatology 2019:1-7.

20. PA J, JS L, E E, H F, E D, S G, et al. MUC5B Promoter Variant and Rheumatoid Arthritis with Interstitial Lung Disease. The New England journal of medicine 2018;379(23):2209-2219.

21. JA S, X H, J H, EA F, A Z, HM F, et al. Rheumatoid Arthritis Disease Activity Predicting Incident Clinically Apparent Rheumatoid Arthritis-Associated Interstitial Lung Disease: A Prospective Cohort Study. Arthritis \& rheumatology (Hoboken, N.J.) 2019;71(9):1472-1482.

22. GE F, K C. Lung cancer in rheumatoid arthritis. Is there a need for better risk assessment and screening? Clinical rheumatology 2020;39(3):957961.

23. FA P, DC S, El S. A Study of Multiple Causes of Death in Rheumatoid Arthritis. The Journal of rheumatology 2015;42(12):2221-8.

\section{Figures}



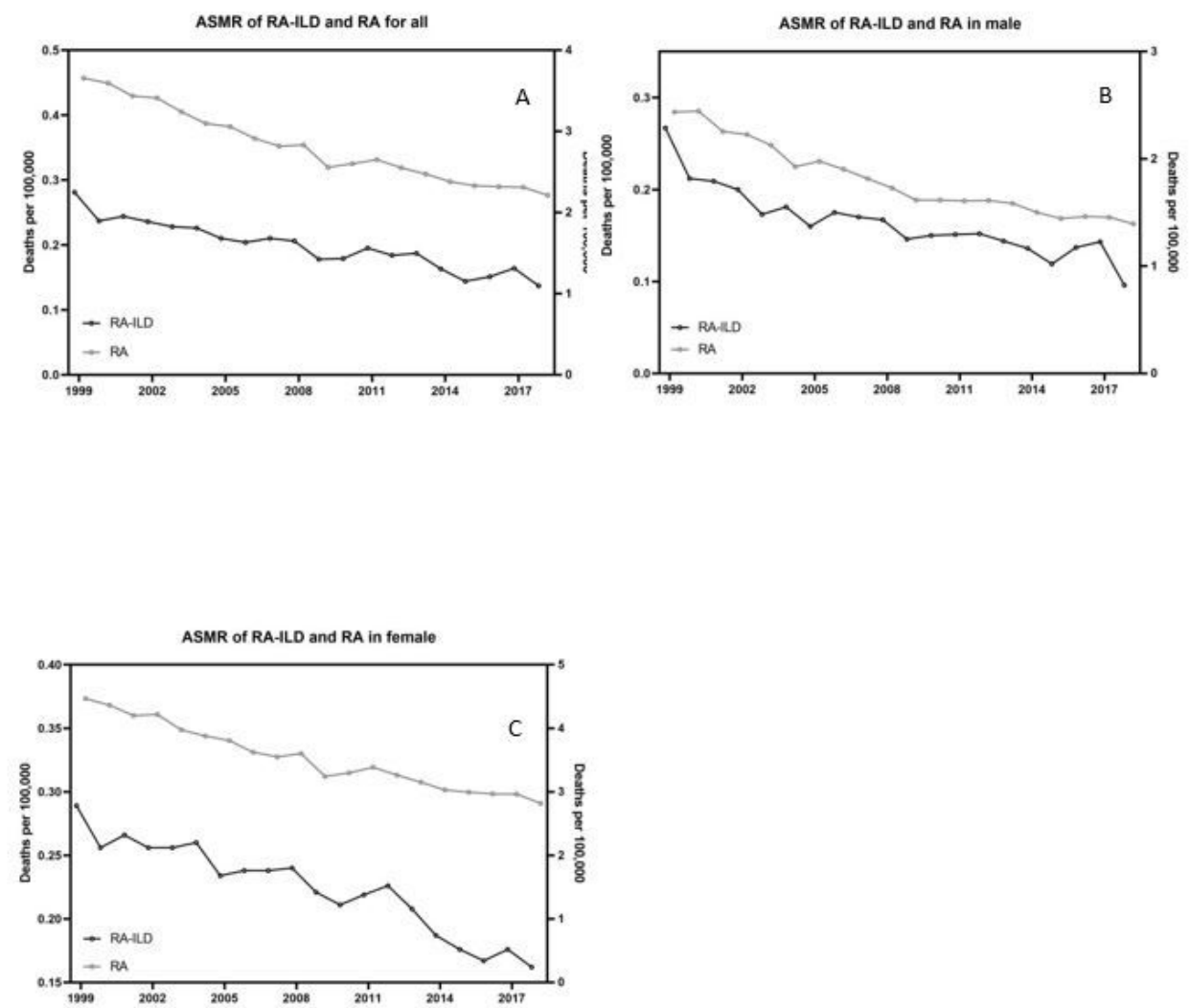

\section{Figure 1}

Age-standardised mortality rates for rheumatoid arthritis (RA) and RA with interstitial lung disease (RA-ILD), overall and among males and females, 1999-2018. The data of the RA-ILD group is referred to in the left $Y$ axis, and that of RA is referred to in the right $Y$ axis. 

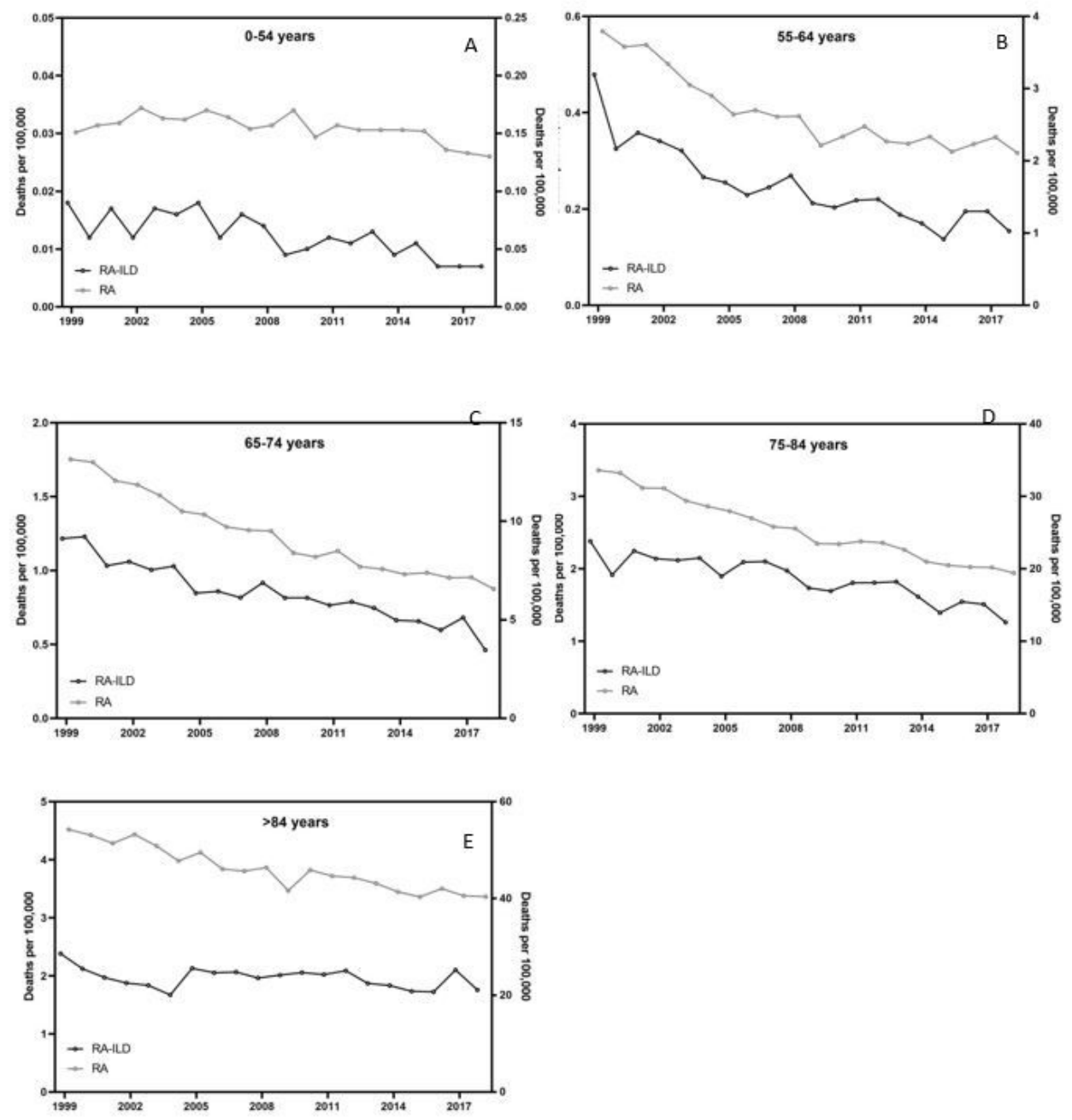

Figure 2

Trends in mortality rates of rheumatoid arthritis (RA) with and without interstitial lung disease (ILD) mentioned in death certificates by age group $₫ 0-54$, 55-64, 65-74, and 75-84years $囚$ in the U.S., 1999-2018. The data of the RA-ILD group is referred to in the left Y axis, and that of RA is referred to in the right $Y$ axis. 